\title{
ANALYSIS OF THE HYDROSTATIC APPROXIMATION IN OCEANOGRAPHY WITH COMPRESSION TERM*
}

\author{
Tomás Chacón Rebollo ${ }^{1}$, Roger Lewandowski ${ }^{2}$ and Eliseo Chacón Vera ${ }^{1}$
}

\begin{abstract}
The hydrostatic approximation of the incompressible 3D stationary Navier-Stokes equations is widely used in oceanography and other applied sciences. It appears through a limit process due to the anisotropy of the domain in use, an ocean, and it is usually studied as such. We consider in this paper an equivalent formulation to this hydrostatic approximation that includes Coriolis force and an additional pressure term that comes from taking into account the pressure in the state equation for the density. It therefore models a slight dependence of the density upon compression terms. We study this model as an independent mathematical object and prove an existence theorem by means of a mixed variational formulation. The proof uses a family of finite element spaces to discretize the problem coupled with a limit process that yields the solution. We finish this paper with an existence and uniqueness result for the evolutionary linear problem associated to this model. This problem includes the same additional pressure term and Coriolis force.
\end{abstract}

Mathematics Subject Classification. 35Q30, 76D05.

Received: November 5, 1998. Revised: October 7, 1999.

\section{INTRODUCTION}

The anisotropic stationary Navier-Stokes equations are widely used in geophysical fluid dynamics as a mathematical model for water flow in lakes and oceans, see for instance [10,11]. These equations appear when the domain in use has very different horizontal and vertical dimensions, the turbulent viscosity coefficients may not be isotropic in this case. When we consider a shallow domain, i.e. one in which the horizontal dimension is very large compared with the vertical one, the hydrostatic approximation is the basic model. This model is obtained through a limit process from the anisotropic stationary Navier-Stokes equations and it is studied as such by Besson and Laydi in [2] and by Bresch and Simon in [3]. Several codes have also been developed to solve this problem [15].

In this paper we study the stationary nonlinear hydrostatic approximation for the ocean as an independent mathematical object. We generalize the linear model that was first considered by Lewandowsky in $[8,9]$. A severe constraint on the Coriolis force and other parameters was required in the study of this model. We do not need this constraint in our analysis.

\footnotetext{
Keywords and phrases. Navier-Stokes equations, Oceanography, Compression term.

* Dedicated to J.L. Lions in honor to his 70th Birthday.

${ }^{1}$ Departamento de Ecuaciones Diferenciales y Análisis Numérico Universidad de Sevilla, 41.080-Sevilla, Spain.

e-mail: chacon@numer2.us.es; eliseo@numer2.us.es

2 Modal-X, Bât. G, Université Paris X, 200 avenue de la République, 92001 Nanterre, France.

e-mail: lewandow@modalx.u-paris10.fr
} 
Our main innovation from the physical point of view is to include in this model the pressure in the state equation for the density, as introduced in Appendix A of [8]. From the mathematical point of view, this modification yields an additional pressure term in the momentum equation which prevents the pressure from taking its usual role as a Lagrange multiplier. A fixed point argument coupled with a standard discretization to this problem by appropriate finite element spaces gives a weak solution to our model. Such approximation turns out to be a useful technical tool for two reasons: it provides an easy way to regularize the problem and a convergent numerical approximation. Our existence theorem is at the same time a convergence result for this approximation.

\subsection{The model equations}

We first introduce the necessary notation for what follows. Let $\Omega \subset \mathbb{R}^{3}$ be the domain defined by

$$
\Omega=\left\{(x, y, z) \in \mathbb{R}^{3} ; \text { s.t. }(x, y) \in S,-D(x, y)<z<0\right\}
$$

where $S \subset \mathbb{R}^{2}$ is an open and bounded Lipschitz domain representing the surface of the ocean and $D: S \cup \partial S \mapsto \mathbb{R}$ is a bounded positive Lipschitz function that describes the bottom and sidewalls of the ocean $\Omega$, denoted by $\Gamma_{b}$ and $\Gamma_{l}$, i.e.

$$
\begin{aligned}
& \Gamma_{b}=\left\{(x, y, z) \in \mathbb{R}^{3} \text { s.t. }(x, y) \in S, z=-D(x, y)\right\}, \\
& \Gamma_{l}=\left\{(x, y, z) \in \mathbb{R}^{3} \text { s.t. }(x, y) \in \partial S,-D(x, y)<z<0\right\},
\end{aligned}
$$

and such that there exists a real number $\delta$ with

$$
\min _{(x, y) \in S \cup \partial S} D(x, y) \geq \delta>0
$$

As we will deal with an anisotropic problem we consider the operator $\nabla_{*}=\left(\partial_{x}, \partial_{y}\right)$, while the notations $\nabla$ and $\Delta$ will keep their standard $3 D$ meaning. We use the operator $\Delta_{\vec{\nu}}$ defined by $\Delta_{\vec{\nu}}=\nu \partial_{x x}^{2}+\nu \partial_{y y}^{2}+\nu_{z} \partial_{z z}^{2}$, where $\vec{\nu}=\left(\nu, \nu, \nu_{z}\right)$ is a turbulent cinematic viscosity vector with $\nu \neq \nu_{z}$, both positive numbers.

The model we present is the following one: Find a velocity field $\vec{u}=\left(\vec{u}_{\star}, u_{3}\right): \Omega \mapsto \mathbb{R}^{3}$, where $\vec{u}_{\star}=\left(u_{1}, u_{2}\right)$, and a scalar function, called superficial pressure, $\pi_{S}: S \mapsto \mathbb{R}$ such that

$$
\begin{aligned}
& (\vec{u} \cdot \nabla) \vec{u}_{\star}-\Delta_{\vec{\nu}} \vec{u}_{\star}+\alpha \vec{u}_{\star}^{\perp}+\frac{1}{\rho_{0}}(1-\gamma z) \nabla_{*} \pi_{S}=\overrightarrow{\mathbf{f}} \quad \text { in } \quad \Omega \\
& \nabla \cdot \vec{u}=0 \quad \text { in } \Omega, \\
& \nu_{z} \partial_{z} \vec{u}_{\star}=\overrightarrow{\mathbf{g}}_{S} \quad \text { on } S \text {, } \\
& \vec{u}_{\star}=0 \quad \text { on } \quad \Gamma_{b} \cup \Gamma_{l}, \\
& u_{3} n_{3}=0 \quad \text { on } \partial \Omega \text {. }
\end{aligned}
$$

In these equations $\overrightarrow{\mathbf{f}}$ is a source term and $\overrightarrow{\mathbf{g}}_{S}$ is the wind tension on the surface $S$. The Coriolis force is given by the term $\alpha \vec{u}_{\star}^{\perp}=\alpha\left(u_{2},-u_{1}\right)$, where $\alpha$ and $\vec{\nu}$ are positive fixed numbers and $\gamma$ is a positive number that will be properly chosen later on. The outward normal vector to the domain $\Omega$ is given by $\vec{n}=\left(n_{1}, n_{2}, n_{3}\right)$ and $\rho_{0}$ denotes a reference value for the density.

Let us justify formally our model. The dimensionless anisotropic stationary Navier-Stokes equations for an incompressible fluid with Coriolis forces in a domain $\Omega$ seek a velocity $\vec{u}=\left(\vec{u}_{\star}, u_{3}\right): \Omega \mapsto \mathbb{R}^{3}$ and a pressure 
$P: \Omega \mapsto \mathbb{R}$, that solve the equations

$$
\begin{aligned}
& (\vec{u} \cdot \nabla) \vec{u}_{\star}-\Delta_{\vec{\nu}} \vec{u}_{\star}+\alpha \vec{u}_{\star}{ }^{\perp}+\frac{1}{\rho_{0}} \nabla_{*} P=\overrightarrow{\mathbf{f}} \quad \text { in } \quad \Omega, \\
& \epsilon^{2}\left\{(\vec{u} \cdot \nabla) u_{3}+\Delta_{\vec{\nu}} u_{3}\right\}+\epsilon u_{1} h+\frac{1}{\rho} \partial_{z} P=-1 \quad \text { in } \Omega, \\
& \nabla \cdot \vec{u}=0 \quad \text { in } \quad \Omega \\
& \nu_{z} \partial_{z} \vec{u}_{\star}=\overrightarrow{\mathbf{g}}_{S} \quad \text { on } \quad S \\
& \vec{u}=0 \quad \text { on } \quad \Gamma_{b} \cup \Gamma_{l} \\
& u_{3}=0 \quad \text { on } \quad S
\end{aligned}
$$

where $\epsilon$ is the ratio depth of the ocean over the diameter of the surface, $\rho$ is the density, $\rho_{0}$ being a reference density, the gravity constant is taken to be one and $h$ is a regular and bounded function.

Equations (1.10-1.15) take into account the Boussinesq approximation, i.e. we assume a constant value for the density in the horizontal momentum and continuity equations. In particular, this approximation implies the incompressibility of the $3 D$ velocity field $\vec{u}$. Also, the boundary condition (1.13) and the fact that $u_{3}=0$ on $S$ constitute what is known as Rigid Lid Hypothesis: We assume no vertical movement of the ocean surface and include in condition (1.13) the wind and turbulence effect on the surface and low atmosphere. This surface $S$ is an "averaged surface" ( $\approx 100$ metres thick) that models the turbulent mixed layer, air-ocean. The information of this mixed layer is given by the boundary condition at $S$.

When we neglect first and second order terms in $\epsilon$ we obtain the hydrostatic approximation: Find $\vec{u}=\left(\vec{u}_{\star}, u_{3}\right): \Omega \mapsto \mathbb{R}^{3}$ and a hydrostatic pressure $P: \Omega \mapsto \mathbb{R}$, such that

$$
\begin{aligned}
& (\vec{u} \cdot \nabla) \vec{u}_{\star}-\Delta_{\vec{\nu}} \vec{u}_{\star}+\alpha \vec{u}_{\star}^{\perp}+\frac{1}{\rho_{0}} \nabla_{*} P=\overrightarrow{\mathbf{f}} \quad \text { in } \quad \Omega, \\
& \frac{1}{\rho} \partial_{z} P=-1 \quad \text { in } \Omega \text {, } \\
& \nabla \cdot \vec{u}=0 \quad \text { in } \Omega \text {, } \\
& \nu \partial_{z} \vec{u}_{\star}=\overrightarrow{\mathbf{g}}_{S} \text { on } S \text {, } \\
& \vec{u}=0 \quad \text { on } \quad \Gamma_{b} \cup \Gamma_{l}, \\
& u_{3} n_{3}=0 \quad \text { on } \quad \partial \Omega \text {. }
\end{aligned}
$$

In [2] it is proved that, when $\rho$ is constant, equations $(1.16-1.21)$ are the limit equations of $(1.10-1.15)$ in a precise sense.

A more realistic model allows a state equation for the density $\rho$ of the form

$$
\rho=\rho(\hat{S}, T, P)
$$

where $\hat{S}$ is the salinity, $T$ is temperature and $P$ is pressure. The dependence of $\rho$ on these variables is mainly polynomial, as experimental examples show in [7]. The functions $\hat{S}$ and $T$ do not add any new mathematical difficulty and, hence, are not considered in our model. But, when we consider $\rho=\rho(P)$ we may argue as follows:

$$
\rho(P)=\rho\left(\pi_{S}+z \partial_{z} \hat{P}\right)=\rho\left(\pi_{S}\right)+z \partial_{z} P(\hat{\theta}) \rho^{\prime}(\tilde{P})
$$

where $\pi_{S}=P(x, y, 0)$ is the pressure at height $z=0$, and $\hat{\theta}$ and $\tilde{P}$ are intermediate values. The superficial pressure $\pi_{S}$ is not the hydrostatic pressure on $S$. Its introduction is a consequence of the Rigid Lid Hypothesis 
and allows to recover the hydrostatic pressure on $\Omega$. Equation (1.11) reads now

$$
\epsilon^{2} \rho\left\{(\vec{u} \cdot \nabla) u_{3}+\nu \Delta u_{3}\right\}+\epsilon u_{1} h+\partial_{z} P=-\rho\left(\pi_{S}\right)-z \partial_{z} P(\hat{\theta}) \rho^{\prime}(\tilde{P}) .
$$

We now may assume a weak dependence of $\rho$ with respect to the pressure $P$, and, therefore, suppose that $z \rho^{\prime}(\tilde{P})$ is small. This hypothesis agrees with the small physical variation of the density around its reference value $\rho_{0}$. Then, a first approximation leads to the simplification of (1.24)

$$
\partial_{z} P=-\rho\left(\pi_{S}\right)
$$

and, from here,

$$
P=\pi_{S}+\int_{z}^{0} \rho\left(\pi_{S}\right) \mathrm{d} \xi
$$

Hence, a linear dependence of $\rho$ from $\pi_{S}$ gives a law of the form

$$
\rho\left(\pi_{S}\right)=\rho_{0}+\gamma\left(\pi_{S}-\pi_{S}^{0}\right)
$$

for some positive number $\gamma$ and a superficial pressure of reference $\pi_{S}{ }^{0}$. Finally, we eliminate the hydrostatic $3 D$ pressure $P$ in the momentum equation for the horizontal velocity $\vec{u}_{\star}=\left(u_{1}, u_{2}\right)$, using the relation

$$
\nabla_{*} P=(1-\gamma z) \nabla_{*} \pi_{S}
$$

Therefore, we obtain model (1.5-1.9) from the hydrostatic approximation (1.16-1.21) and we see that equations (1.5-1.9) intend to model a slight dependence of the density upon compression effects. We refer the reader to [8] for a more detailed account on the physical interpretations of this problem.

The main result presented in this paper states that for $\gamma$ small enough there exists a weak solution $\left(\vec{u}, \pi_{S}\right)$ to model (1.5-1.9), see Theorem 2, Section 1.3, for a detailed statement.

Remark 1. As we mentioned before, the most general model also includes equations for temperature and salinity coupled with the momentum equation. From the mathematical point of view, these two functions do not add any new difficulty. The essential difficulties of this problem are the lack of regularity of the vertical velocity $u_{3}$ in the non-linear convection term and the additional compression term.

\subsection{Basic functional spaces and related results}

On the domain $\Omega$ introduced in the previous section we consider the following linear space of smooth functions

$$
C_{b, l}^{\infty}=\left\{\phi \in C^{\infty}(\bar{\Omega}) \quad \text { s.t. } \quad \phi_{\left.\right|_{\Gamma_{b}} \cup \Gamma_{l}}=0\right\},
$$

and, based on this, the Hilbert space

$$
H_{b, l}^{1}(\Omega)={\overline{C_{b, l}^{\infty}}}^{\left(H^{1}(\Omega)\right)}=\left\{v \in H^{1}(\Omega) \quad \text { s.t. } \quad v_{\left.\right|_{\Gamma_{b} \cap \Gamma_{l}}}=0\right\}
$$

endowed with the standard $H_{0}^{1}(\Omega)$ norm. In general, for any integer $k \geq 1$ and real $p \geq 1$ we may consider the spaces

$$
W_{b, l}^{k, p}(\Omega)={\overline{C_{b, l}^{\infty}}}^{\left(W^{k, p}(\Omega)\right)}=\left\{v \in W^{k, p}(\Omega) \quad \text { s.t. } \quad v_{\left.\right|_{\Gamma_{b} \cap \Gamma_{l}}}=0\right\}
$$


endowed with the $W^{k, p}(\Omega)$ norm. We will work with product spaces and use the notation $\mathbf{H}_{b, l}^{1}(\Omega)=H_{b, l}^{1}(\Omega) \times$ $H_{b, l}^{1}(\Omega)$ and $\mathbf{W}_{b, l}^{k, p}(\Omega)=W_{b, l}^{k, p}(\Omega) \times W_{b, l}^{k, p}(\Omega)$. Trace spaces will also be needed, we introduce

$$
H^{1 / 2}(\partial \Omega)=\left\{v \in L^{2}(\partial \Omega) \quad \text { s.t. } \quad v=\bar{v}_{\mid \partial \Omega} \quad \text { for some } \bar{u} \in H^{1}(\Omega)\right\},
$$

and the anisotropic spaces:

$$
\begin{aligned}
& H\left(\partial_{z} ; \Omega\right)=\left\{v \in L^{2}(\Omega) \quad \text { s.t. } \quad \partial_{z} v \in L^{2}(\Omega)\right\}, \\
& H_{0}\left(\partial_{z} ; \Omega\right)=\bar{C}_{0}^{\infty}(\Omega) \\
& H\left(\partial_{z} ; \Omega\right)
\end{aligned}
$$

endowed with the norm $\|v\|_{H\left(\partial_{z} ; \Omega\right)}=\|v\|_{L^{2}(\Omega)}+\left\|\partial_{z} v\right\|_{L^{2}(\Omega)}$. As usual, we denote by $U^{*}$ the dual space of a Banach space $U$ and, in particular, we consider the dual spaces $H^{-1 / 2}(\partial \Omega)=\left(H^{1 / 2}(\partial \Omega)\right)^{\star}, \quad \mathbf{H}^{-1 / 2}=$ $\left(H^{-1 / 2}(\partial \Omega)\right)^{2}$ and $\mathbf{H}_{b, l}^{-1}=\left(\mathbf{H}_{b, l}^{1}(\Omega)\right)^{*}$. The normal trace $v n_{3}$ of functions in $H\left(\partial_{z} ; \Omega\right)$ belongs to $H^{-1 / 2}(\partial \Omega)$ and $H_{0}\left(\partial_{z} ; \Omega\right)$ is described as

$$
H_{0}\left(\partial_{z} ; \Omega\right)=\left\{v \in H\left(\partial_{z} ; \Omega\right) \text { s.t. } v n_{\left.3\right|_{\partial \Omega}}=0\right\}
$$

where $\overrightarrow{\mathbf{n}}=\left(n_{1}, n_{2}, n_{3}\right)$ denotes the outward normal to $\Omega$ (see [12] for all these results). We also introduce, for any $r \geq 1$, the Banach space $U_{r}$ given by

$$
U_{r}=\left\{v \in H_{b, l}^{1}(\Omega) \quad \text { s.t. } \quad \partial_{z} v \in L^{r}(\Omega)\right\}
$$

endowed with the norm $\|v\|_{U_{r}}=|v|_{1,0}+\left\|\partial_{z} v\right\|_{L^{r}(\Omega)}$ and the product space $\mathbf{U}_{r}=U_{r} \times U_{r}$. Finally, we consider

$$
L_{0}^{2}(S)=\left\{\pi: S \mapsto \mathbb{R} \quad \text { s.t. } \quad\|\pi\|_{0, S}^{2}=\int_{S}|\pi|^{2} \mathrm{~d} S<+\infty, \int_{S} \pi \mathrm{d} S=0\right\}
$$

normed by $\|\cdot\|_{0, S}$ as usual.

\subsection{Reduction of the model equations and variational formulation}

In this section we first introduce the variational formulation of model (1.5-1.9). Then we present a reformulation of (1.5-1.9) in which the vertical velocity $u_{3}$ disappears as an unknown. We finally show that both formulations have the same weak solutions.

The lack of smoothness in the vertical component of the velocity field $u_{3}$ only gives a convection term $u_{3} \partial_{z} \vec{u}_{\star} \in\left(L^{1}(\Omega)\right)^{2}$. The following result gives a variational sense to this term:

Lemma 1. If $\vec{u}_{\star}=\left(u_{1}, u_{2}\right) \in \mathbf{H}_{b, l}^{1}(\Omega)$ and $u_{3} \in H_{0}\left(\partial_{z} ; \Omega\right)$ are such that $\nabla \cdot\left(\vec{u}_{\star}, u_{3}\right)=0$, then, for any $r \geq 3$, $u_{3} \partial_{z} \vec{u}_{\star}$ belongs to $\mathbf{U}_{r}{ }^{\star}$ and

$$
\left\langle u_{3} \partial_{z} \vec{u}_{\star}, \vec{v}_{\star}\right\rangle_{U_{r}^{\star}, U_{r}}=-\int_{\Omega} u_{3} \partial_{z} \vec{v}_{\star} \cdot \vec{u}_{\star} \mathrm{d} \Omega-\int_{\Omega} \partial_{z} u_{3} \vec{v}_{\star} \cdot \vec{u}_{\star} \mathrm{d} \Omega
$$

for all $\vec{v}_{\star} \in \mathbf{U}_{r}$. As a consequence, $\left(\left(\vec{u}_{\star}, u_{3}\right) \cdot \nabla\right) \vec{u}_{\star}$ belongs to $\mathbf{U}_{r}{ }^{\star}$ and

$$
\left\langle\left(\left(\vec{u}_{\star}, u_{3}\right) \cdot \nabla\right) \vec{u}_{\star}, \vec{v}_{\star}\right\rangle_{U_{r}^{\star}, U_{r}}=-\int_{\Omega}\left(\vec{u}_{\star} \cdot \nabla_{*}\right) \vec{v}_{\star} \cdot \vec{u}_{\star} \mathrm{d} \Omega-\int_{\Omega} u_{3} \partial_{z} \vec{v}_{\star} \cdot \vec{u}_{\star} \mathrm{d} \Omega
$$

for all $\vec{v}_{\star} \in \mathbf{U}_{r}$.

Proof. The proof of (1.38) is a consequence of a density argument for approximating $u_{3} \partial_{z} \vec{u}_{\star}$, coupled with an integration by parts, while (1.39) uses integration by parts and (1.38). 
As the cinematic viscosity vector $\vec{\nu}=\left(\nu, \nu, \nu_{z}\right)$ does not add any mathematical difficulty to the formulation, we will simplify the model and suppose $\nu_{z}=\nu>0$. The variational formulation of problem (1.5-1.9) is the following:

Given $\overrightarrow{\mathbf{f}} \in \mathbf{H}_{b, l}^{-1}$ and $\overrightarrow{\mathbf{g}}_{S} \in \mathbf{H}^{-1 / 2}$, obtain $\left(\vec{u}, \pi_{S}\right) \in H_{b, l}^{1}(\Omega) \times H_{b, l}^{1}(\Omega) \times H_{0}\left(\partial_{z} ; \Omega\right) \times L_{0}^{2}(S), \vec{u}=\left(\vec{u}_{\star}, u_{3}\right)$, such that

$$
\begin{aligned}
\left\langle(\vec{u} \cdot \nabla) \vec{u}_{\star}, \vec{v}_{\star}\right\rangle_{U_{4}^{\star}, U_{4}}+\nu\left(\nabla \vec{u}_{\star}, \nabla \vec{v}_{\star}\right)_{\Omega}+\alpha\left(\vec{u}_{\star}^{\perp}, \vec{v}_{\star}\right)_{\Omega}-\frac{1}{\rho_{0}}\left(\pi_{S},(1-\gamma z) \nabla_{*} \cdot \vec{v}_{\star}\right)_{\Omega}=l\left(\vec{v}_{\star}\right), \\
(q, \nabla \cdot \vec{u})_{\Omega}=0,
\end{aligned}
$$

for all $\left(\vec{v}_{\star}, q\right) \in \mathbf{U}_{4} \times L_{0}^{2}(\Omega)$. Here $l \in \mathbf{H}_{b, l}^{-1}$ is the linear form $l: \mathbf{H}_{b, l}^{1}(\Omega) \mapsto \mathbb{R}$ defined by

$$
l\left(\vec{v}_{\star}\right)=\left\langle\overrightarrow{\mathbf{f}}, \vec{v}_{\star}\right\rangle_{\Omega}+\left\langle\overrightarrow{\mathbf{g}}_{S}, \vec{v}_{\left.\star\right|_{S}}\right\rangle_{S},
$$

where $\langle\cdot, \cdot\rangle_{\Omega}$ and $\langle\cdot, \cdot\rangle_{S}$ stand for the duality in $H^{1}(\Omega)$ and $H^{1 / 2}(S)$ respectively, and $(\cdot, \cdot)_{\Omega}$ stand for the $L^{2}$ scalar, or tensor, product in $\Omega$. Any solution of (1.40-1.41) is a weak solution of problem (1.5-1.9) in the following sense: equation (1.5) is verified in $\mathbf{U}_{4}{ }^{\star}$, equation (1.6) in $L^{2}(\Omega),(1.7)$ in $\mathbf{H}^{-1 / 2},(1.8)$ in $H^{1 / 2}\left(\Gamma_{b} \cup \Gamma_{l}\right)$ and (1.9) in $H^{-1 / 2}(\partial \Omega)$.

Remark 2. The choice of test functions in $\mathbf{U}_{4}$ is due to the lack of regularity in the vertical velocity $u_{3}$ and it just makes computations easier. The simplest choice, $\mathbf{U}_{3}$, yields some technical trouble in the compactness argument used later on. Moreover, it essentially gives the same information on the solution.

Let us now consider the following reduced problem: Find a velocity field $\vec{u}_{\star}: \Omega \mapsto \mathbb{R}^{2}$ and a superficial pressure $\pi_{S}: S \mapsto \mathbb{R}$ such that

$$
\begin{aligned}
& \left(\vec{u}_{\star}, u_{3}\right) \cdot\left(\nabla_{*}, \partial_{z}\right) \vec{u}_{\star}-\nu \Delta \vec{u}_{\star}+\alpha \vec{u}_{\star}{ }^{\perp}+\frac{1}{\rho_{0}}(1-\gamma z) \nabla_{*} \pi_{S}=\overrightarrow{\mathbf{f}} \quad \text { in } \Omega, \\
& \nabla_{*} \cdot\left\langle\vec{u}_{\star}\right\rangle=0 \quad \text { on } S, \\
& \nu \partial_{z} \vec{u}_{\star}=\overrightarrow{\mathbf{g}}_{S} \quad \text { on } S \text {, } \\
& \vec{u}_{\star}=0 \quad \text { on } \quad \Gamma_{b} \cup \Gamma_{l}, \\
& \int_{z}^{0} \nabla_{*} \cdot \vec{u}_{\star}(x, y, \xi) \mathrm{d} \xi=u_{3}(x, y, z) \text { in } \Omega .
\end{aligned}
$$

Here, for any function $\phi: \Omega \mapsto \mathbb{R}$ we will denote by $\langle\cdot\rangle$ its vertical mean, i.e.

$$
\langle\phi\rangle(x, y)=\int_{-D(x, y)}^{0} \phi(x, y, z) \mathrm{d} z, \quad \forall(x, y) \in S .
$$

In order to obtain a variational formulation of (1.43-1.46) we consider the functional $B:\left(\mathbf{H}_{b, l}^{1}(\Omega) \times L_{0}^{2}(S)\right) \times$ $\left(\mathbf{U}_{4} \times L_{0}^{2}(S)\right) \mapsto \mathbb{R}$ given by

$$
\begin{aligned}
B\left(\left(\vec{u}_{\star}, \pi_{S}\right) ;\left(\vec{v}_{\star}, q\right)\right)= & \left\langle(\vec{u} \cdot \nabla) \vec{u}_{\star}, \vec{v}_{\star}\right\rangle_{U_{4}^{\star}, U_{4}}+\nu\left(\nabla \vec{u}_{\star}, \nabla \vec{v}_{\star}\right)_{\Omega}-\alpha\left(\vec{u}_{\star}^{\perp}, \vec{v}_{\star}\right)_{\Omega}-\frac{1}{\rho_{0}}\left(\pi_{S}, \nabla_{*} \cdot\left\langle\vec{v}_{\star}\right\rangle\right)_{S} \\
& +\frac{1}{\rho_{0}}\left(\pi_{S},\left\langle\gamma z \nabla_{*} \cdot \vec{v}_{\star}\right\rangle\right)_{S}-\left(q, \nabla_{*} \cdot\left\langle\vec{u}_{\star}\right\rangle\right)_{S},
\end{aligned}
$$

where $(\cdot, \cdot)_{S}$ stand for the $L^{2}$ scalar product in $S$ and $u_{3}$ is given by (1.47). 
We pose the following variational problem:

Given $\overrightarrow{\mathbf{f}} \in \mathbf{H}_{b, l}^{-1}, \overrightarrow{\mathbf{g}}_{S} \in \mathbf{H}^{-1 / 2}$ and positive constants $\gamma, \nu$ and $\alpha$, find $\left(\vec{u}_{\star}, \pi_{S}\right) \in \mathbf{H}_{b, l}^{1}(\Omega) \times L_{0}^{2}(S)$ such that

$$
B\left(\left(\vec{u}_{\star}, \pi_{S}\right) ;\left(\vec{v}_{\star}, q\right)\right)=l\left(\vec{v}_{\star}\right), \quad \forall\left(\vec{v}_{\star}, q\right) \in \mathbf{U}_{4} \times L_{0}^{2}(S) .
$$

The variational formulations $(1.50)$ and $(1.40,1.41)$ are equivalent due to the following result:

Lemma 2. Let $\vec{u}_{\star} \in \mathbf{H}_{b, l}^{1}(\Omega)$ and define $u_{3}$ by

$$
u_{3}(x, y, z)=\int_{z}^{0} \nabla_{*} \cdot \vec{u}_{\star}(x, y, \xi) \mathrm{d} \xi, \quad \forall(x, y, z) \in \Omega .
$$

Then, the following are satisfied:

1. We have $u_{3} \in H\left(\partial_{z} ; \Omega\right)$ and $\partial_{z} u_{3}=-\nabla_{*} \cdot \vec{u}_{\star}$.

2. The $3 D$ velocity vector $\left(\vec{u}_{\star}, u_{3}\right)$ is incompressible, i.e., $\nabla \cdot\left(\vec{u}_{\star}, u_{3}\right)=0$ in $L^{2}(\Omega)$.

3. $\left\langle\vec{u}_{\star}\right\rangle(x, y)=\int_{-D(x, y)}^{0} \vec{u}_{\star}(x, y, z) \mathrm{d} z \in H_{0}^{1}(S)$.

4. $u_{3} n_{3 \mid \partial \Omega} \in H^{-1 / 2}(\partial \Omega)$ and

$$
u_{3} n_{\left.3\right|_{\partial \Omega}}=0 \quad \text { in } \quad H^{-1 / 2}(\partial \Omega) \Longleftrightarrow \nabla_{*} \cdot\left\langle\vec{u}_{\star}\right\rangle=0 \quad \text { in } \quad S,
$$

where $\overrightarrow{\mathbf{n}}=\left(n_{1}, n_{2}, n_{3}\right)$ is the $3 D$ outward normal vector to $\partial \Omega$. As a consequence of (1.44), we obtain $u_{3} \in H_{0}\left(\partial_{z} ; \Omega\right)$.

Proof. The first two properties are straightforward while the third one is obtained by a density argument. We prove the fourth one: As $\vec{u}_{\star_{\Gamma_{b}} \cup \Gamma_{l}}=0$ and $D$ is bounded and Lipschitz, we have $D \in W^{1, \infty}(S)$ and then

$$
\nabla_{*} \cdot\left\langle\vec{u}_{\star}\right\rangle=\left\langle\nabla_{*} \cdot \vec{u}_{\star}\right\rangle
$$

for all $\vec{u}_{\star} \in \mathbf{H}_{b, l}^{1}(\Omega)$. Now, assume that $u_{3} n_{\left.3\right|_{\partial \Omega}}=0$ in $H^{-1 / 2}(\partial \Omega)$, then any $\varphi \in C_{0}^{\infty}(S)$ may be considered as a $z$-independent function in $\Omega$, and we have

$$
\begin{aligned}
\int_{S} \nabla_{*} \cdot\left\langle\vec{u}_{\star}\right\rangle \varphi \mathrm{d} S & =\int_{\Omega} \nabla_{*} \cdot \vec{u}_{\star} \varphi \mathrm{d} \Omega=-\int_{\Omega} \partial_{z} u_{3} \varphi \mathrm{d} \Omega \\
& =\int_{\Omega} u_{3} \partial_{z} \varphi \mathrm{d} \Omega-\int_{\partial \Omega}\left(u_{3} n_{3}\right) \varphi \mathrm{d} \partial \Omega \\
& =-\left\langle u_{3} n_{3}, \varphi_{\mid \partial \Omega}\right\rangle=0 .
\end{aligned}
$$

Thus, $\nabla_{*} \cdot\left\langle\vec{u}_{\star}\right\rangle=0$ in $S$.

Now we suppose that $\nabla_{*} \cdot\left\langle\vec{u}_{\star}\right\rangle=0$, then take a sequence $\vec{\phi}_{n} \in C_{b, l}^{\infty} \times C_{b, l}^{\infty}$ convergent to $\vec{u}_{\star}$ in $\left(H^{1}(\Omega)\right)^{2}$ and consider $\Phi_{n}=\int_{z}^{0} \nabla_{*} \cdot \overrightarrow{\phi_{n}} \mathrm{~d} \xi$. As $\Phi_{n}$ converges to $u_{3}$ in $H\left(\partial_{z} ; \Omega\right), \Phi_{n} n_{3}$ converges to $u_{3} n_{3}$ in $H^{-1 / 2}(\partial \Omega)$. We see next that, indeed, $\Phi_{n} n_{3}$ converges to 0 in $H^{-1 / 2}(\partial \Omega)$. As we have $\Phi(x, y, 0)=0$ and $n_{3}=0$ on $S \cup \Gamma_{l}$, then, for any $v \in H^{1 / 2}(\partial \Omega)$

$$
\begin{aligned}
\left|\left\langle\Phi_{n} n_{3}, v\right\rangle_{H^{1 / 2}(\partial \Omega)}\right|= & \left|\int_{\partial \Omega} \Phi_{n} n_{3} v \mathrm{~d} \partial \Omega\right|=\left|\int_{\Gamma_{b}} \Phi_{n} n_{3} v \mathrm{~d} \partial \Omega\right| \\
= & \left|\int_{S}\left(\Phi_{n} n_{3} v\right)(x, y,-D(x, y)) \sqrt{1+|\nabla D|^{2}} \mathrm{~d} x \mathrm{~d} y\right| \\
& \leq C(D, S)\left\|\Phi_{n}(\cdot,-D(\cdot))\right\|_{L^{2}(S)}\|v\|_{L^{2}(S)} \\
& \leq C(D, S)\left\|\nabla_{*} \cdot\left\langle\overrightarrow{\phi_{n}}\right\rangle\right\|_{L^{2}(S)}|v|_{L^{2}(S)}
\end{aligned}
$$


where $C(D, S)$ is a constant that depends only on $\Omega$. We conclude now thanks to the fact that $\Phi_{n}(x, y,-D(x, y))$ converges to zero in $L^{2}(S)$ because $\nabla_{*} \cdot\left\langle\overrightarrow{\phi_{n}}\right\rangle$ converges to $\nabla_{*} \cdot\left\langle\vec{u}_{\star}\right\rangle=0$ in $L^{2}(S)$.

Therefore, we get the following equivalence result:

Theorem 1. Given positive numbers $\vec{\nu}, \alpha$ and $\gamma,\left(\vec{u}, \pi_{S}\right) \in H_{b, l}^{1}(\Omega) \times H_{b, l}^{1}(\Omega) \times H_{0}\left(\partial_{z} ; \Omega\right) \times L_{0}^{2}(S)$, with $\vec{u}=$ $\left(\vec{u}_{\star}, u_{3}\right)$, is a solution of (1.40-1.41) if and only if $\left(\vec{u}_{\star}, \pi_{S}\right) \in \mathbf{H}_{b, l}^{1}(\Omega) \times L_{0}^{2}(S)$ solves (1.50), with $u_{3}=\int_{z}^{0} \nabla_{*}$. $\vec{u}_{\star} \mathrm{d} \xi$.

Remark 3. Observe that with the choice of test functions made all the terms in the two variational formulations introduced are well defined.

The existence result of weak solution to problem (1.5-1.9) that we present in this paper is the following:

Theorem 2. Let $\overrightarrow{\mathbf{f}} \in \mathbf{H}_{b, l}^{-1}, \overrightarrow{\mathbf{g}}_{S} \in \mathbf{H}^{-1 / 2}$ and positive numbers $\vec{\nu}, \alpha$ and $\gamma$. Suppose that $D \in W^{1, \infty}(S)$ is such that (1.4) is satisfied. Then, there exists a positive constant $C$ depending only on the data such that, if $\gamma<C$ there exists a pair $\left(\vec{u}_{\star}, \pi_{S}\right) \in \mathbf{H}_{b, l}^{1}(\Omega) \times L_{0}^{2}(S)$ that satisfies the variational problem (1.50).

Remark 4. Hypothesis (1.4) is needed for technical reasons, as it will be mentioned later on.

Remark 5. It is possible to work with a more general function $\gamma=\gamma(z) \in L^{\infty}(\Omega)$ such that $\gamma(z) \neq \gamma z$. Then we just require $\|\gamma\|_{\infty}$ to be small enough.

Remark 6. The uniqueness of the solution for problem (1.50) is still an open question due to the lack of regularity in the third component of the velocity field, which leads to the non-Hilbertian weak formulation presented in this paper.

\section{Proof of TheOrem 2}

\subsection{Sketch of the proof and preliminaries}

We outline the proof of Theorem 2:

1. We consider a family of finite dimensional spaces $\mathbf{X}_{h} \times M_{h}$ dense in both $\mathbf{H}_{b, l}^{1}(\Omega) \times L_{0}^{2}(S)$ and $\mathbf{U}_{4} \times L_{0}^{2}(S)$, depending upon the norm used and then we pose the variational problem (1.50) in $\left(\mathbf{X}_{h} \times M_{h}\right) \times\left(\mathbf{X}_{h} \times M_{h}\right)$. Observe that the convection term is regularized with this discretization of the problem.

2. We then remove the nonlinearity in the convection term and in the pressure term by means of a known data $(\overrightarrow{\mathbf{a}}, t) \in \mathbf{X}_{h} \times M_{h}$.

3. We show existence of solution $\left(\vec{u}_{\star}, \pi_{S}\right) \in \mathbf{X}_{h} \times M_{h}$ for this linear problem.

4. We estimate this solution in terms of the data $(\overrightarrow{\mathbf{a}}, t)$.

5. We show existence of solution, by a fixed point argument, for the non-linear and with additional pressure term problem in the finite-dimensional space $\mathbf{X}_{h} \times M_{h}$.

6. Via a compactness argument, we obtain the solution $\left(\vec{u}_{\star}, \pi_{S}\right) \in \mathbf{H}_{b, l}^{1}(\Omega) \times L_{0}^{2}(S)$ to (1.50) by passing to the limit in the equation.

We perform an approximation of the domain $\Omega$ through polygonal subdomains $\Omega_{h} \subset \Omega$, for $h>0$, as follows: Take the the surface of $\Omega_{h}$ to be a polygonal domain $S_{h}$ and consider a triangularization of $S_{h}$. Take a Lipschitz function $D_{h}: S_{h} \rightarrow \mathbb{R}$ that gives the depth of the domain $\Omega_{h}$, as in the continuous case, such that the extension of $D_{h}$ by zero to converges to $D$ in $W^{1, \infty}(S)$ and

$$
\begin{aligned}
\min _{(x, y) \in S_{h}} D_{h}(x, y) & \geq \delta>0, \\
\left\|D_{h}\right\|_{1, \infty} & \leq C\|D\|_{1, \infty},
\end{aligned}
$$

for $C$ and $\delta$ independent of $h$. We recall that $\delta$ is the one in (1.4). Then we form $\Omega_{h}$ by vertical prisms spanned from the triangularization of $S_{h}$ down to the bottom and such that the depth is given by $D_{h}$. Then, when $h$ tends to $0, \Omega_{h}$ fills up $\Omega$. 
We follow [5] and consider $\mathbf{Y}_{h}$ and $\tilde{M}_{h}$ finite element subspaces of $\left(W_{0}^{1, \infty}\left(S_{h}\right)\right)^{2}$ and $L_{0}^{\infty}\left(S_{h}\right)$ respectively, such that the discrete "inf-sup" condition by Ladyzhenskya-Brezzi-Babuška, the LBB condition, is satisfied, i.e. there exists a constant $\hat{c}>0$, independent of $h$, with

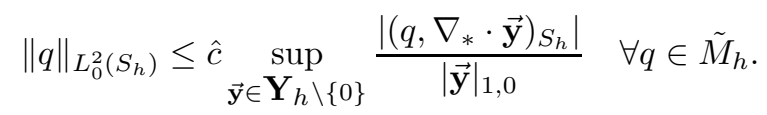

We approximate $\mathbf{H}_{b, l}^{1}(\Omega)$ by a family of finite element subspaces $\tilde{\mathbf{Y}}_{h}$ on $\Omega_{h}$, such that $\tilde{\mathbf{Y}}_{h} \subset\left(W_{b, l}^{1, \infty}\left(\Omega_{h}\right)\right)^{2}$ and form a "stable extension" of the spaces $\mathbf{Y}_{h}$ in the sense that the following conditions are satisfied. Consider the nonzero function

$$
\beta(z)=\left\{\begin{array}{ll}
\delta^{-1}(z+\delta), & \text { for }-\delta \leq z \leq 0 \\
0, & \text { for } z \leq-\delta
\end{array} .\right.
$$

We then require:

- (H1) for all $\overrightarrow{\mathbf{y}} \in \mathbf{Y}_{h}$

$$
\beta(z) \overrightarrow{\mathbf{y}}(x, y) \in \tilde{\mathbf{Y}}_{h} .
$$

- (H2) The union of spaces $\bigcup_{h>0} \mathbf{X}_{h}$ and $\bigcup_{h>0} M_{h}$ are dense in $\mathbf{U}_{4}$ and $L_{0}^{2}(S)$ respectively, where $\mathbf{X}_{h}$ and $M_{h}$ are the extension by zero of functions in $\tilde{\mathbf{Y}}_{h}$ and $\tilde{M}_{h}$.

As $\Omega_{h}$ has been constructed, these properties are simply obtained via the standard Lagrange finite elements, see for instance [6] (page 89). In particular (1.4) plays a key role in (H1). From now on we will work with the spaces $\mathbf{X}_{h}$ and $M_{h}$.

We now pose the finite dimensional version of the non-linear variational problem (1.50) and solve it in the next section:

Find $\left(\vec{u}_{\star}, \pi_{S}\right) \in \mathbf{X}_{h} \times M_{h}$ such that

$$
B_{h}\left(\left(\vec{u}_{\star}, \pi_{S}\right) ;\left(\vec{v}_{\star}, q\right)\right)=l_{h}\left(\vec{v}_{\star}\right), \quad \forall\left(\vec{v}_{\star}, q\right) \in \mathbf{X}_{h} \times M_{h}
$$

where $B_{h}$ and $l_{h}$ denote the restriction of $B$ to $\mathbf{X}_{h} \times M_{h}$ and of $l$ to $\mathbf{X}_{h}$ respectively.

\subsection{Existence of solution for the finite dimensional linear problem}

We first make problem (2.57) into a linear problem that yields a square linear system of dimension $\operatorname{dim}\left(\mathbf{X}_{h}\right)+$ $\operatorname{dim}\left(M_{h}\right)$. Consider a known velocity field $\overrightarrow{\mathbf{a}} \in \mathbf{X}_{h}$, and consider $b(x, y, z)=\int_{z}^{0} \nabla_{*} \cdot \overrightarrow{\mathbf{a}}(x, y, \xi) \mathrm{d} \xi$, for $(x, y, z) \in \Omega_{h}$ and $t \in M_{h}$, then we pose the following finite dimensional linear problem:

Find $\left(\vec{u}_{\star}, \pi_{S}\right) \in \mathbf{X}_{h} \times M_{h}$ such that

$$
B_{h, \overrightarrow{\mathbf{a}}}\left(\left(\vec{u}_{\star}, \pi_{S}\right) ;\left(\vec{v}_{\star}, q\right)\right)=l_{h, t}\left(\vec{v}_{\star}\right), \quad \forall\left(\vec{v}_{\star}, q\right) \in \mathbf{X}_{h} \times M_{h}
$$

where $B_{h, \overrightarrow{\mathbf{a}}}:\left(\mathbf{X}_{h} \times M_{h}\right) \times\left(\mathbf{X}_{h} \times M_{h}\right) \mapsto \mathbb{R}$ is the linear form given by

$$
\begin{aligned}
B_{h, \overrightarrow{\mathbf{a}}}\left(\left(\vec{u}_{\star}, \pi_{S}\right) ;\left(\vec{v}_{\star}, q\right)\right)= & \left(\left(\overrightarrow{\mathbf{a}} \cdot \nabla_{*}\right) \vec{u}_{\star}, \vec{v}_{\star}\right)+\left(b \partial_{z} \vec{u}_{\star}, \vec{v}_{\star}\right)+\nu\left(\nabla \vec{u}_{\star}, \nabla \vec{v}_{\star}\right)+\alpha\left(\vec{u}_{\star}{ }^{\perp}, \vec{v}_{\star}\right) \\
& -\left(\pi_{S}, \nabla_{*} \cdot\left\langle\vec{v}_{\star}\right\rangle\right)+\left(q, \nabla_{*} \cdot\left\langle\vec{u}_{\star}\right\rangle\right),
\end{aligned}
$$

and the linear form $l_{h, t}: \mathbf{X}_{h} \mapsto \mathbb{R}$ is defined by

$$
l_{h, t}\left(\vec{v}_{\star}\right)=\left\langle\overrightarrow{\mathbf{f}}, \vec{v}_{\star}\right\rangle_{\Omega}+\left\langle\overrightarrow{\mathbf{g}}_{S}, \vec{v}_{\star}\right\rangle_{S}-\left(t, \gamma\left\langle z \nabla_{*} \cdot \vec{v}_{\star}\right\rangle\right)_{S} .
$$

We have the following result: 
Lemma 3. Given $\overrightarrow{\mathbf{f}} \in \mathbf{H}_{b, l}^{-1}, \overrightarrow{\mathbf{g}}_{S} \in \mathbf{H}^{-1 / 2}$ and $D \in W^{1, \infty}(S)$ satisfying (1.4), there exists a unique solution $\left(\vec{u}_{\star}, \pi_{S}\right) \in \mathbf{X}_{h} \times M_{h}$ to the linear finite dimensional problem (2.58) such that the following bounds hold

$$
\begin{aligned}
\left|\vec{u}_{\star}\right|_{1,0} & \leq \frac{C}{\nu}\left\{\|\overrightarrow{\mathbf{f}}\|_{-1}+\left\|\overrightarrow{\mathbf{g}}_{S}\right\|_{-1 / 2}+\gamma\|t\|_{0, S}\right\}, \\
\left\|\pi_{S}\right\|_{0, S} & \leq \frac{C}{\delta^{7 / 4}}\left\{\left(|\overrightarrow{\mathbf{a}}|_{1,0}+\nu+\alpha\right)\left|\vec{u}_{\star}\right|_{1,0}+\|\overrightarrow{\mathbf{f}}\|_{-1}+\left\|\overrightarrow{\mathbf{g}}_{S}\right\|_{-\frac{1}{2}}+\gamma\|t\|_{0, S}\right\},
\end{aligned}
$$

where $C$ is a constant that depends only on the domain $\Omega$, not on $h$, and $\delta$ is given in (1.4).

Remark 7. Observe that this $L^{2}$ estimate for the pressure blows up when $\delta$ goes to zero.

Proof. As we have a finite dimensional problem that yields a square linear system of equations, we will obtain existence and uniqueness of solution if we prove that the homogeneous problem just have the trivial solution. Therefore, bounds of the possible solution in terms of the right hand side $l_{h, t}$ would be enough for existence and uniqueness. Let us prove $(2.61,2.62)$ :

Let $\left(\vec{u}_{\star}, \pi_{S}\right) \in \mathbf{X}_{h} \times M_{h}$ be a solution of (2.58), then using as a test function the pair $\left(\vec{u}_{\star},-\pi_{S}\right)$ and the fact that $\nabla \cdot(\overrightarrow{\mathbf{a}}, b)=0$ we obtain

$$
\begin{aligned}
\nu\left|\vec{u}_{\star}\right|_{1,0}^{2}= & \left\langle\overrightarrow{\mathbf{f}}, \vec{u}_{\star}\right\rangle_{\Omega}+\left\langle\overrightarrow{\mathbf{g}}_{S}, \vec{u}_{\left.\star\right|_{S_{h}}}\right\rangle_{S}-\left(t,\left\langle\gamma z \nabla_{*} \cdot \vec{u}_{\star}\right\rangle\right)_{S} \\
& \leq\|\overrightarrow{\mathbf{f}}\|_{-1}\left|\vec{u}_{\star}\right|_{1,0}+C\left\|\overrightarrow{\mathbf{g}}_{S}\right\|_{H^{-1 / 2}}\left|\vec{u}_{\star}\right|_{1,0}+\gamma\|t\|_{0, S}\|D\|_{\infty}^{1 / 2}\left\|\nabla_{*} \cdot \vec{u}_{\star}\right\|_{0, \Omega} \\
& \leq C\left\{\|\overrightarrow{\mathbf{f}}\|_{-1}+\left\|\overrightarrow{\mathbf{g}}_{S}\right\|_{-1 / 2}+\gamma\|t\|_{0, S_{h}}\right\}\left|\vec{u}_{\star}\right|_{1,0}
\end{aligned}
$$

and (2.61) follows.

In order to obtain (2.62), we will make use of the LBB condition (2.54) that the pair of spaces $\left(\mathbf{Y}_{h}, \tilde{M}_{h}\right)$ satisfies. Given $\vec{v}_{\star} \in \mathbf{Y}_{h}$ we let $\overrightarrow{\mathbf{w}} \in \mathbf{X}_{h}$ be given by

$$
\overrightarrow{\mathbf{w}}(x, y, z)=\beta(z) \vec{v}_{\star}(x, y), \quad \forall(x, y, z) \in \Omega_{h},
$$

and extended by zero outside $\Omega_{h}$, where the function $\beta$ is given in (H1). We easily obtain

$$
\begin{aligned}
\langle\overrightarrow{\mathbf{w}}\rangle(x, y) & =\frac{\delta}{2} \vec{v}_{\star}(x, y) \quad \forall(x, y) \in S, \\
\|\overrightarrow{\mathbf{w}}\|_{U_{4}} & \leq C \delta^{-3 / 4}\left|\vec{v}_{\star}\right|_{1,0},
\end{aligned}
$$

for $C$ independent of $h$. Now we use as a test function the pair $(\overrightarrow{\mathbf{w}}, 0)$ and obtain

$$
\begin{aligned}
\frac{\delta}{2}\left(\pi_{S}, \nabla_{*} \cdot \vec{v}_{\star}\right)_{S}= & \left(\left(\overrightarrow{\mathbf{a}} \cdot \nabla_{*}\right) \vec{u}_{\star}, \overrightarrow{\mathbf{w}}\right)+\left(b \partial_{z} \vec{u}_{\star}, \overrightarrow{\mathbf{w}}\right)+\nu\left(\nabla \vec{u}_{\star}, \nabla \overrightarrow{\mathbf{w}}\right)+\alpha\left(\vec{u}_{\star}{ }^{\perp}, \overrightarrow{\mathbf{w}}\right) \\
& -\langle\overrightarrow{\mathbf{f}}, \overrightarrow{\mathbf{w}}\rangle_{\Omega}+\left\langle\overrightarrow{\mathbf{g}}_{S}, \overrightarrow{\mathbf{w}}\right\rangle_{S} \\
& -\left(t,\left\langle\gamma z \nabla_{*} \cdot \overrightarrow{\mathbf{w}}\right\rangle\right)_{S} .
\end{aligned}
$$

Therefore,

$$
\left|\left(\pi_{S}, \nabla_{*} \cdot \vec{v}_{\star}\right)_{S}\right| \leq \frac{C}{\delta^{7 / 4}}\left\{\left(|\overrightarrow{\mathbf{a}}|_{1,0}+\nu+\alpha\right)\left|\vec{u}_{\star}\right|_{1,0}+\|\overrightarrow{\mathbf{f}}\|_{-1}+\left\|\overrightarrow{\mathbf{g}}_{S}\right\|_{-\frac{1}{2}}+\gamma\|t\|_{0, S}\right\}\left\|\vec{v}_{\star}\right\|_{1,0},
$$

and, finally, the LBB condition (2.54) yields (2.62). 


\subsection{Existence of solution for the finite dimensional non-linear problem}

Now we use the result in Lemma 3 combined with a fixed point argument to obtain the solution of the non-linear problem (2.57).

Consider the mapping $T: \mathbf{X}_{h} \times M_{h} \mapsto \mathbf{X}_{h} \times M_{h}$ defined by $T((\overrightarrow{\mathbf{a}}, t))=\left(\vec{u}_{\star}, \pi_{S}\right)$, where the pair $\left(\vec{u}_{\star}, \pi_{S}\right)$ is the unique solution of the linear problem in $\mathbf{X}_{h} \times M_{h}$ obtained with data $(\overrightarrow{\mathbf{a}}, t)$. We have:

Lemma 4. The following are satisfied:

1. The operator $T$ is continuous and bounded.

2. If $\gamma<C$ for some positive real number $C$ that depends only on the domain $\Omega$ and all the known data, then $T$ has a fixed point $\left(\vec{u}_{\star}, \pi_{S}\right) \in \mathbf{X}_{h} \times M_{h}$ that solves the non-linear variational problem in $\mathbf{X}_{h} \times M_{h}$.

Proof. Continuity of $T$ is an easy consequence of the facts that we work in finite dimension and of the uniqueness of solution in problem (2.58), while boundeness of $T$ is given by Lemma 3 . We check now the fixed point condition for $T$. Suppose that we have two positive constants $A_{u}$ and $A_{p}$ such that

$$
\begin{aligned}
|\overrightarrow{\mathbf{a}}|_{1,0} & \leq A_{u}, \\
\|t\|_{L_{0}^{2}(S)} & \leq A_{p} .
\end{aligned}
$$

We will show that $T$ maps the product of discs $D\left(0, A_{u}\right) \times D\left(0, A_{p}\right)$ of $\mathbf{X}_{h} \times M_{h}$ into itself. For this purpose we just have to consider the bounds given by Lemma 3 ,

$$
\begin{aligned}
\left|\vec{u}_{\star}\right|_{1,0} & \leq \frac{C}{\nu}\left\{\|\overrightarrow{\mathbf{f}}\|_{-1}+\left\|\overrightarrow{\mathbf{g}}_{S}\right\|_{-1 / 2}+\gamma\|t\|_{0, S}\right\}, \\
\left\|\pi_{S}\right\|_{0, S} & \leq \frac{C}{\delta^{7 / 4}}\left\{\left(|\overrightarrow{\mathbf{a}}|_{1,0}+\nu+\alpha\right)\left|\vec{u}_{\star}\right|_{1,0}+\|\overrightarrow{\mathbf{f}}\|_{-1}+\left\|\overrightarrow{\mathbf{g}}_{S}\right\|_{-1 / 2}+\gamma\|t\|_{0, S}\right\},
\end{aligned}
$$

and find two positive constants $A_{u}, A_{p}$ such that

$$
\begin{aligned}
\frac{C}{\nu}\left\{\|\overrightarrow{\mathbf{f}}\|_{-1}+\left\|\overrightarrow{\mathbf{g}}_{S}\right\|_{-1 / 2}+\gamma A_{p}\right\} & =A_{u} \\
\frac{C}{\delta^{7 / 4}}\left\{\left(A_{u}+\nu+\alpha\right) A_{u}+\|\overrightarrow{\mathbf{f}}\|_{-1}+\left\|\overrightarrow{\mathbf{g}}_{S}\right\|_{-1 / 2}+\gamma A_{p}\right\} & =A_{p} .
\end{aligned}
$$

For this purpose, we substitute (2.70) into (2.71) to obtain a second order polynomial equation in $A_{p}$ that must have a positive root:

$$
b_{0} A_{p}^{2}+b_{1} A_{p}+b_{2}=0
$$

Here, the coefficients read as follows:

$$
\begin{aligned}
& b_{0}=\frac{C^{3}}{\delta^{7 / 4} \nu^{2}} \gamma^{2} \\
& b_{1}=\frac{1}{\delta^{7 / 4} \nu^{2}}\left\{C^{3} \tau^{2}+C^{2}(C \tau+\nu(\nu+\alpha)\} \gamma-1\right. \\
& b_{2}=\frac{\tau C}{\delta^{7 / 4} \nu^{2}}\left\{\nu^{2}+C(C \tau+\nu(\nu+\alpha)\}\right.
\end{aligned}
$$

where $\tau=\|\overrightarrow{\mathbf{f}}\|_{-1}+\left\|\overrightarrow{\mathbf{g}}_{S}\right\|_{-1 / 2}$ and $C$ only depends on $\Omega$. We just need to find $A_{p}>0$ such that (2.72) is satisfied. A simple algebra yields a condition of the form

$$
\gamma<\Phi(\nu, \alpha, \tau) \delta^{7 / 4}
$$


for some positive function $\Phi$ bounded in $\nu$ and decreasing in $\tau$ and $\alpha$. Therefore, for $\gamma$ small enough, $T$ has a fixed point $\left(\vec{u}_{\star}, \pi_{S}\right) \in D\left(0, A_{u}\right) \times D\left(0, A_{p}\right)$ such that

$$
B_{h}\left(\left(\vec{u}_{\star}, \pi_{S}\right) ;\left(\vec{v}_{\star}, q\right)\right)=l_{h}\left(\vec{v}_{\star}\right), \quad \forall\left(\vec{v}_{\star}, q\right) \in \mathbf{X}_{h} \times M_{h} .
$$

\subsection{Convergence analysis}

We now perform the convergence analysis that leads to a weak solution of (1.50) by a standard compactness argument.

By the finite dimensional analysis performed in the previous section, there exists a family of solutions $\left(\vec{u}_{\star h}, \pi_{S h}\right) \in \mathbf{X}_{h} \times M_{h}$ of problem (2.77). As these functions are defined in $\Omega_{h}$ and $S_{h}$, we consider their extension by zero and work on the fixed domains $\Omega$ and $S$. Then, these solutions are bounded in $\mathbf{H}_{b, l}^{1}(\Omega) \times L_{0}^{2}(S)$. Therefore, there exists a subsequence of solutions, denoted in the same way, which is weakly convergent to a pair $\left(\vec{u}_{\star}, \pi_{S}\right) \in \mathbf{H}_{b, l}^{1}(\Omega) \times L_{0}^{2}(S)$ as $h$ decreases to 0 . We show next that this pair is a solution of (1.50). Due to (H2), for each pair $\left(\vec{v}_{\star}, q\right) \in \mathbf{U}_{4} \times L_{0}^{2}(S)$, we can find a sequence $\left(\vec{\phi}_{h}, q_{h}\right) \in \mathbf{X}_{h} \times M_{h}$ strongly convergent to $\left(\vec{v}_{\star}, q\right)$ in $\mathbf{U}_{4} \times L_{0}^{2}(S)$. We, then, write (1.50) for $\left(\vec{u}_{\star h}, \pi_{S h}\right)$ and $\left(\vec{\phi}_{h}, q_{h}\right)$ in $\mathbf{X}_{h} \times M_{h}$ and take limits when $h$ decreases to 0 in each term. The only term that gives some difficulty is the one coming from the convection term and which involves the vertical component of the velocity, $u_{3}$ :

$$
\left(u_{3}^{h} \partial_{z} \vec{\phi}_{h}, \vec{u}_{\star}^{h}\right)_{\Omega} .
$$

The reason is that $u_{3}$ only belongs to $H_{0}\left(\partial_{z}, \Omega\right)$. But the weak convergence of $u_{3}^{h}$ to $u_{3}$ in $L^{2}(\Omega)$, the strong convergence of $\partial_{z} \vec{\phi}_{h}$ to $\partial_{z} \vec{v}_{\star}$ in $L^{4}(\Omega)$ and the strong convergence of $\vec{u}_{\star h}$ to $\vec{u}_{\star}$ in $L^{4}(\Omega)$, obtained by the compact injection of $H^{1}(\Omega)$ into $L^{4}(\Omega)$, insure the existence of the limit. The proof of Theorem 2 is completed.

Remark 8. Hypothesis (1.4) seems to be essential to obtain uniform, i.e., independent of $h$, bounds for discrete velocity and pressure in the norm $H^{1}(\Omega) \times L^{2}(S)$. This fact allows the limit process in this problem with additional pressure term.

Remark 9. With the technique presented in this paper we may obtain, for a right hand side in $H^{-1}$ and without the additional pressure term, a velocity in $H^{1}(\Omega)$ and a pressure in $L^{3 / 2}(\Omega)$, as in [2]. In this case condition (1.4) is not needed.

\section{The LineAr EVOLUtion CASE}

In this final section we continue the study of the hydrostatic approximation and go a step further to the linear evolution problem. The model is the following: Find a velocity field $\vec{u}_{\star}: \Omega \times(0, T) \mapsto \mathbb{R}^{2}$ and a scalar function $\pi_{S}: S \times(0, T) \mapsto \mathbb{R}$ such that

$$
\begin{aligned}
\frac{\partial \vec{u}_{\star}}{\partial t}-\nu \Delta \vec{u}_{\star}+\alpha \vec{u}_{\star}{ }^{\perp}+(1-\gamma z) \nabla_{*} \pi_{S} & =\overrightarrow{\mathbf{f}} \quad \text { in } \Omega, \\
\nabla_{*} \cdot\left\langle\vec{u}_{\star}\right\rangle & =0 \text { in } S, \\
\nu \partial_{z} \vec{u}_{\star} & =\overrightarrow{\mathbf{g}}_{S} \text { in } S, \\
\vec{u}_{\left.\star\right|_{\Gamma_{b}} \cup \Gamma_{l}} & =0,
\end{aligned}
$$

is satisfied in some sense. Here $\gamma, \nu$ and $\alpha$ are positive constants and $\overrightarrow{\mathbf{f}}, \overrightarrow{\mathbf{g}}_{S}$ play the same role as before.

We will keep the hypothesis (1.4) on the function $D$ which describes the bottom of the domain $\Omega$. It implies $D \in W^{1, \infty}(S)$ and $D^{-1} \in L^{\infty}(S)$. Beside the already introduced functional spaces, we consider the following 
nonzero linear space of smooth functions

$$
\mathcal{F}=\left\{\vec{\phi} \in C_{b, l}^{\infty} \times C_{b, l}^{\infty} \quad \text { s.t. } \quad \nabla_{*} \cdot\langle\vec{\phi}\rangle(x, y)=0 \quad \forall(x, y) \in S\right\},
$$

and the following Hilbert space, see [8]:

$$
\mathbf{V}=\overline{\mathcal{F}}^{\left(\left(H^{1}(\Omega)\right)^{2}\right)}=\left\{\vec{v} \in \mathbf{H}_{b, l}^{1}(\Omega) \quad \text { s.t. } \quad \nabla_{*} \cdot\langle\vec{v}\rangle=0 \quad \text { in } \quad S\right\}
$$

with the standard $\left(H_{0}^{1}(\Omega)\right)^{2}$ norm. We will also use $\mathbf{H}=\overline{\mathcal{F}}^{\left(\left(L^{2}(\Omega)\right)^{2}\right)}$ endowed with the $L^{2}$ norm and the dual space of $\mathbf{V}$, denoted by $\mathbf{V}^{\star}$.

We state the following existence and uniqueness result for $(3.79,3.82)$ :

Theorem 3. Let $\overrightarrow{\mathbf{f}} \in L^{2}(0, T ; \mathbf{H}), \overrightarrow{\mathbf{g}}_{S} \in L^{2}\left(0, T ; \mathbf{H}^{-1 / 2}\right)$ with $\frac{\mathrm{d}}{\mathrm{d} t} \overrightarrow{\mathbf{g}}_{S} \in L^{2}\left(0, T ; \mathbf{H}^{-1 / 2}\right)$, suppose that $\vec{u}_{\star 0} \in \mathbf{V}$ and let $\alpha, \nu$ be positive constants. Then, there exists a positive number $C>0$, depending on the domain $\Omega$ and the known data, such that when $\gamma<C$ there exists a unique function $\vec{u}_{\star} \in L^{2}(0, T ; \mathbf{V})$ with $\frac{\mathrm{d}}{\mathrm{d} t} \vec{u}_{\star} \in$ $L^{2}(0, T ; \mathbf{V}) \cap L^{2}(0, T ; \mathbf{H})$ and a superficial pressure $\pi_{S} \in L^{2}\left(0, T ; L_{0}^{2}(S)\right)$ such that (3.79-3.82) is satisfied in $L^{2}\left(0, T ; \mathbf{H}_{b, l}^{-1}\right)$.

The proof of this result is based on three points; the standard Galerkin techniques used for the linear evolution Stokes problem, see mainly [13,14], a modification of De Rham's Lemma, see [12] for instance, obtained in [8] (page 43), and a fixed point argument as in the previous Sections. The additional smoothness required on $\overrightarrow{\mathbf{g}}_{S}$ is needed to estimate the time derivative of $\vec{u}_{\star}$.

As for the hydrostatic approximation to the nonstationary Navier-Stokes equations, see $[1,4]$ for the model without compression term, we could not apply this fixed point technique for the compression term due to the lack of regularity in the time variable for the superficial pressure. It, therefore, remains as an open problem.

\section{REFERENCES}

[1] Azerat P. and Guillén F., Équations de Navier-Stokes en bassin peu profond: l'approximation hydrostatique. Submitted for publication.

[2] Besson O. and Laydi M.R., Some Estimates for the Anisotropic Navier-Stokes Equations and for the Hydrostatic Approximation. RAIRO Modél. Math. Anal. Numér. 26 (1992) 855-865.

[3] Bresch D. and Simon J., Modèles stationnaires de lacs et mers. Équations aux dérivées partielles et applications. Articles dédiés à J.-L. Lions. Elsevier, Paris (1998).

[4] Bresch D., Lemoine J. and Simon J., Écoulement engendré par le vent et la force de Coriolis dans un domain mince: II cas d'évolution. C. R. Acad. Sci. Paris Sér. I 327 (1998) 329-334.

[5] Bravo de Mansilla A., Chacón Rebollo T. and Lewandowski R., Observaciones sobre dos aplicaciones diversas del Método de los Elementos Finitos: Controlabilidad Exacta de la Ecuación Discreta del Calor y Ecuaciones Primitivas en Oceanografía, Actas de la Jornada Científica en Homenaje al Prof. Antonio Valle Sánchez (1997).

[6] Ciarlet Ph., The Finite Element Method for Elliptic Problems. North-Holland (1978).

[7] Gill A.-E., Atmosphere-Ocean dynamics. Academic Press (1982).

[8] Lewandowski R., Analyse mathématique et océanographie. Masson (1997).

[9] Lewandowski R., Étude d'un système stationnaire linéarisé d'équations primitives avec des termes de pression additionnels. C. R. Acad. Sci. Paris Sér. I 324 (1997) 173-178.

[10] Lions J.-L., Teman S. and Wang S., On the equations of the large scale Ocean. Nonlinearity 5 (1992) 1007-1053.

[11] Pedlosky J., Geophysical fluid dynamics. Springer-Verlag, New York (1987).

[12] Teman R., Sur la stabilité et la convergence de la méthode des pas fractionnaires. Ann. Mat. Pura ed Applicata LXXIX (1968) 191-379.

[13] Teman R., Navier-Stokes Equations. North-Holland, Elsevier (1985).

[14] Zeidler E., Nonlinear functional analysis and its applications, II/A. Springer-Verlag (1990).

[15] Zuur E. and Dietrich D.E., The SOMS model and its application to Lake Neuchâtel. Aquatic Sci. 52 (1990) 115-129. 